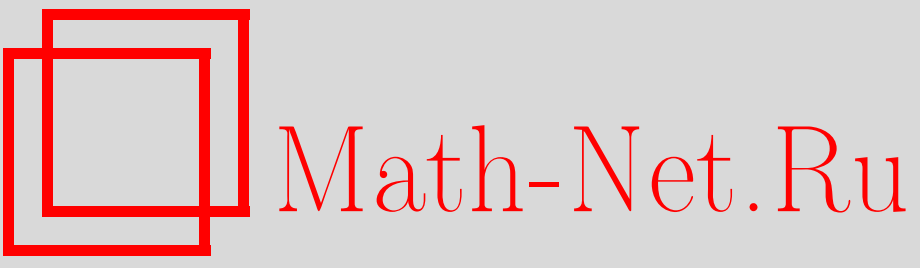

Р. Т. Курдиани, Когомологии Хохшильда и расширения, УМH, 2005, том 60, выпуск 5, 169-170

DOI: https://doi.org/10.4213/rm1648

Использование Общероссийского математического портала Math-Net.Ru подразумевает, что вы прочитали и согласны с пользовательским соглашением

http://www.mathnet.ru/rus/agreement

Параметры загрузки:

IP : 54.237 .206 .68

26 апреля 2023 г., 06:19:01 


\title{
КОГОМОЛОГИИ ХОХШИЛЬДА И РАСШИРЕНИЯ
}

\author{
Р. Т. КуРдИАнИ
}

В данной статье $n$-я группа когомологий Хохшилда (определение см. в [1]) описьвается $(n-2)$ расширениями (теорема 1). При $n=2,3$ теорема совпадает с известными классическими результатами. В случае $n=1$ мы получаем описание группы дифференцирований при помощи расширений, а при $n \geqslant 4$ эта теорема дает новое описание групп когомологий.

ОПРЕДЕЛЕниЕ 1 . Пусть $A$ - ассоциативная алгебра и $M$ - некоторый $A-A$-бимодуль. Будем рассматривать $A$ как $D G$-алгебру, имеющую лишь компоненту в размерности 0 и тривиальный дифференциал. Пусть $s^{n} M-D G$-алгебра с тривиальным дифференциалом и тривиальньм произведением, причем $\left(s^{n} M\right)_{n}=M$ и $\left(s^{n} M\right)_{i}=0$ при $i \neq n$. Точная последовательность $D G$-алгебр длины $\leqslant n$

$$
s^{n} M \stackrel{\varepsilon}{\longrightarrow} E \stackrel{\pi}{\longrightarrow} A
$$

называется $n$-расширением $A$ с помощью $M$, если выполнены следующие условия:

а) $\varepsilon(\pi(e) m)=e \varepsilon(m), \varepsilon(m \pi(e))=\varepsilon(m) e$ для любых $e \in E$ и $m \in M$;

б) $\pi(\varepsilon(m))=0$ для любого $m \in M$; из этого условия вытекает, что композиция Cone $(\varepsilon) \stackrel{\text { proj }}{\longrightarrow}$ $E \longrightarrow A$ является цепньм отображением (при $n>0$ это условие следует само собой);

в) $\operatorname{Cone}(\varepsilon) \rightarrow A$ является квазиизоморфизмом.

Расширение называется расщепляемым, если отображение Cone $(\mathrm{Cone}(\varepsilon) \rightarrow A)$ допускает стягивание.

При $n=0$ определенное выше понятие 0-расширения совпадает с хорошо известным понятием расширения ассоциативной алгебры при помощи бимодуля. В явном виде, $n$-расширение при $n>0$ представляет собой точную последовательность

$$
0 \longrightarrow M \longrightarrow E_{n} \longrightarrow \cdots \longrightarrow E_{0} \longrightarrow A \longrightarrow 0,
$$

где $E=\left(E_{n} \rightarrow \cdots \rightarrow E_{0}\right)-D G$-алгебра, отображение $E_{0} \rightarrow A$ вляется отображением ассоциативных алгебр, а отображение $M \rightarrow E_{n}$ является отображением $E_{0}-E_{0}$-бимодулей, где структура $E_{0}-E_{0}$-бимодуля на $M$ индуцирована отображением $E_{0} \rightarrow A$. В таких обозначениях расширение является расщепляемым, если точная последовательность (1) стягиваема.

Определение $n$-расширения имеет смысл также и при $n=-1$. А именно, (-1)-расширение представляет собой диаграмму вида

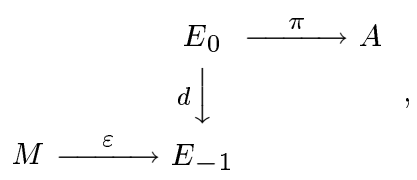

где $E_{0} \rightarrow E_{-1}-D G$-алгебра, $\pi$ - отображение ассоциативных алгебр, $\varepsilon$ - отображение $E_{0}-E_{0}$-бимодулей, а отображение $M \oplus E_{0} \rightarrow E_{-1} \oplus A$, заданное матрицей $\left(\begin{array}{ll}\varepsilon & d \\ 0 & \pi\end{array}\right)$, является изоморфизмом. Из последнего условия вытекает существование отображений $A \rightarrow E_{0}$ (правого обратного к $\pi$ ) и $E_{-1} \rightarrow M$ (левого обратного к $\varepsilon$ ). Определим $d^{\prime}$ как композицию $A \rightarrow E_{0} \rightarrow E_{-1} \rightarrow M$. Тогда $d^{\prime}: A \rightarrow M-$ это $D G$-алгебра (т.е. дифференцирование из $A$ в $M)$ и является ретрактом отображения $E_{0} \rightarrow E_{-1}$. Заметим, что любое $(-1)$-расширение расщепляемо.

Два $n$-расширения $E$ и $E^{\prime}$ эквивалентны, если существуют третье расширение $F$ и отображения $D G$-алгебр $F \rightarrow E$ и $F \rightarrow E^{\prime}$, индуцирующие тождественные отображения на $s^{n} M$ и $A$. Это действителшно является отношением эквивалентности на множестве $n$-расширений $A$ при помощи $M$. Обозначим через $\operatorname{AlgExt}^{n}(A, M)$ множество классов эквивалентности расщепляемых $n$-расширений $A$ при помощи $M$. 
ТЕОРема 2. Имеется взаимно однозначное соответствие между $\operatorname{AlgExt}^{n-2}(A, M)$ и $n$-й группой когомологий Хохшильда $\mathrm{H}^{n}(A, M)$.

При $n=-1$ получаем описание первых когомологий при помощи расширений:

$$
\mathrm{H}^{1}(A, M) \cong \operatorname{AlgExt}^{-1}(A, M),
$$

которое немного отличается от известного описания при помощи группы дифференцирований.

ЗАмечАниЕ 3 . Аналогичный резултат верен и для $D G$-алгебр. Приводимое ниже доказательство с небольшими изменениями проходит и для этого случая.

ДокАЗАТЕЛЬСтво. Случай $n=1$ очевиден, так что предположим, что $n \geqslant 2$. Доказательство распадается на три шага.

Шаг 1. Данному расширению сопоставим коцикл и докажем, что коциклы, сопоставляемые эквивалентным расширениям, отличаются на кограницу. Пусть $M \rightarrow E \rightarrow A$ - расщепляемое $(n-2)$-расширение $A$ с помошью $M$. Нам нужно построить коцикл $f: A^{\otimes n} \rightarrow M$. Для этого мы при помощи индукции по $i$ построим отображения $h_{i}: A^{\otimes i} \rightarrow E_{i-1}, 1 \leqslant i<n$, и $h_{n}: A^{\otimes n} \rightarrow M$, удовлетворяющие соотношению

$$
\begin{aligned}
d h_{i}\left(a_{1} \otimes \cdots \otimes a_{i}\right)= & \sum_{j=1}^{i-1}(-1)^{j+1} h_{i-1}\left(a_{1} \otimes \cdots \otimes a_{j-1} \otimes a_{j} a_{j+1} \otimes a_{j+2} \cdots \otimes a_{i}\right) \\
& +\sum_{j=1}^{i-1}(-1)^{j} h_{j}\left(a_{1} \otimes \cdots \otimes a_{j}\right) h_{i-j}\left(a_{j+1} \otimes \cdots \otimes a_{i}\right),
\end{aligned}
$$

где $d$ представляет собой либо дифференциал в $E$ при $i<n$, либо вложение $M \rightarrow E_{n-2}$ при $i=n$. Тогда $h_{n}-$ нужный нам коцикл. Легко проверить, что коциклы, сопоставляемые эквивалентным расширениям, представляют один класс когомологий.

Шаг 2. Сопоставим расширение данному коциклу и докажем, что расширения, сопоставляемые коциклам, отличающимся на кограницу, эквиваленты. Пусть $f: A^{\otimes n} \rightarrow M$ - коцикл. Мы должны построить расширение. Рассмотрим омега-бар конструкцию $\Omega B(A)$ для $A$. Мы имеем отображение $\Omega B(A) \rightarrow A$, однако $\Omega B(A)$ не имеет конечной длины. Чтобы получить $D G$-алгебру $F$ желаемой длины, можно рассмотреть усеченную алгебру: $F_{i}=(\Omega B(A))_{i}, i<n-1, F_{n-1}=$ $(\Omega B(A))_{n-1} / \operatorname{Im} d_{n}, F_{i}=0, i>n-1$.

Заметим, что $f$ определяет отображение $F_{n-1} \rightarrow M$. Пусть $X$ обозначает копредел диаграммы $M \leftarrow F_{n-1} \rightarrow F_{n-2}$. Тогда $E=\left(0 \rightarrow X \rightarrow F_{n-3} \rightarrow \cdots \rightarrow F_{1} \rightarrow F_{0} \rightarrow 0\right)$ представляет собой $D G$-алгебру длины $n-2$. Необходимое нам расширение $A$ с помощью $M$ есть $E$ вместе с от ображениями $M \rightarrow X$ и $F_{0} \rightarrow A$. Легко видеть, что два коцикла, представляющие один класс когомологий, дают эквивалентные расширения.

Шаг 3. Покажем, что соответствия, установленные в предыдущих шагах, обратны друг к другу. Пусть $f$ - коцикл. Ясно, что коцикл, получаемьй из расширения $M \rightarrow E \rightarrow A$, которое построено по $f$, совпадает с $f$.

Предположим, что $M \rightarrow L \rightarrow A$-расширение и $f$-коцикл, полученный из этого расширения. Отображения $h_{i}: A^{\otimes i} \rightarrow L_{i-1}(1 \leqslant i \leqslant n-1)$ определяют отображения $\alpha_{j}: E_{j} \rightarrow L_{j}(0 \leqslant j \leqslant$ $n-2)$. Из определения $\alpha$ следует, что оно является отображением $D G$-алгебр, индуцирующим тождественные отображения на $A$ и $M$. Теорема доказана.

\section{СПИСОК ЛИТЕРАТУРЫ}

[1] J.-L. Loday. Cyclic Homology. Berlin: Springer-Verlag, 1998. (Grundlehren Math. Wiss. V. 301.)

University of Aberdeen, United Kingdom

E-mail: revas@maths.abdn.ac.uk
Представлено И. А. Дынниковым Принято редколлегией 22.07.2005 